\title{
Impacto do trabalho auxiliado na qualidade de vida do cirurgião- dentista
}

\section{Impact of assisted work on dentist quality of life}

\section{Daniel de Oliveira LEITE}

Mestre em Biopatologia Bucal pela Faculdade de Odontologia de São José dos Campos - UNESP Univ Estadual Paulista - São José dos Campos - SP - Brasil.

\section{Rodrigo Cembranelli de SOUZA}

Cirurgião-Dentista - Formado pela Faculdade de Odontologia de São José dos Campos - UNESP Univ Estadual Paulista - São José dos Campos - SP - Brasil.

\section{Suely Carvalho Mutti NARESSI \\ Denise NICODEMO}

\section{Eliel Soares ORENHA}

Professor Assistente Doutor - Departamento de Odontologia Social e Clínica Infantil - Faculdade de Odontologia de São José dos Campos - UNESP - Univ Estadual Paulista - São José dos Campos SP - Brasil.

\section{Kecimiylla Sayuri KOGA}

Cirurgiã-Dentista - Formada pela Faculdade de Odontologia de São José dos Campos - UNESP Univ Estadual Paulista - São José dos Campos - SP - Brasil.

\begin{abstract}
Resumo
De um modo geral, a utilização de pessoal auxiliar no consultório odontológico tornou-se fundamental para a melhoria na produtividade, influenciando de maneira direta na qualidade do trabalho e na diminuição do estresse e fadiga profissional. A proposta deste trabalho foi avaliar a qualidade de vida de cirurgiões-dentistas (CDs) que trabalham com e sem auxiliares em saúde bucal (ASBs) em consultórios dentários particulares, mediante aplicação do instrumento de medida validado para a população brasileira, Medical Outcomes Study 36 - Item Short Health Survey (SF-36). O questionário é composto por 11 questões e 36 itens, tendo como propósito avaliar 8 domínios divididos em 2 grandes componentes: o físico - que envolve a capacidade funcional, os aspectos físicos, a dor e o estado geral da saúde; e o mental - que abrange a saúde mental, os aspectos emocionais e sociais e a vitalidade. O estudo foi do tipo qualitativo-descritivo e a amostra foi constituída de 60 CDs com no mínimo 10 anos de exercício profissional, de ambos os gêneros - 30 que trabalhavam auxiliados, na categoria de CD auxiliado e 30 que trabalhavam sem auxiliar, constituindo o grupo de CD não auxiliado. Os resultados obtidos com os testes de significância estatística ANOVA e TUCKEY mostram variação estatística com os domínios: capacidade funcional, dor, estado geral de saúde, vitalidade e aspectos sociais. No grupo de CDs estudado, a condição de trabalho auxiliado impacta positivamente a qualidade de vida considerando os domínios capacidade funcional, dor e estado geral de saúde.
\end{abstract}

\section{UNITERMOS}

Odontologia; auxiliares de odontologia; qualidade de vida; impacto psicossocial. 


\section{INTRODUÇÃO}

Segundo a Organização Mundial de Saúde [1], "qualidade de vida é a percepção do indivíduode sua posição na vida, no contexto da cultura e sistema de valores nos quais ele vive e em relaçãoaos seus objetivos, expectativaspadrõese preocupações”Na áreada saúde, em especial na Odontologia, o interesse pelo conceito qualidadede vida é relativamenterecentee decorre,em parte, dos novos paradigmas que têm influenciado as políticas e as práticas do setor nas últimas décadas.

As transformaçõesque vêm ocorrendona Odontologia nos últimos anos, principalmente relacionadas à atuação profissional,têm exigido mudançasde compor tamento por parte dos cirugiões-dentistas,cujo sucesso não dependemais única e exclusivamentede suas habilidadese conhecimentoscientíficos,mas também de sua capacidade de organização, administração e racionalizaçãodo trabalho.Tais fatorespodeminfluenciare comprometer o bem-estar destes profissionais [2,3].

Entre outras inovações, tal evolução provocou a necessidade de utilização de pessoal auxiliar, o que, indubitavelmente, trouxe um enorme e definitivo benefício para a classe odontológica [4]. Dentre as vantagens da incorporação do pessoal auxiliar na clínica odontológica, destacam-se a maior eficiência e otimização do processo de trabalho; o aumento da qualidade técnica e da produtividade; o conforto e segurança agregados ao atendimento dos pacientes; a minimização do custo operacional e a redução do desgaste físico, do estresse e da fadiga do cirurgião-dentista [5-7]. Além disso, a utilização do trabalho auxiliado contribui positivamente na racionalização do trabalho, poupando o cirurgiãodentista do estresse físico e mental, permitindo que sua concentração permaneça apenas na solução do problema do paciente [5].

Uma vez que o exercício da Odontologia abrange um grande número de áreas inter-relacionadas, delegar atividades a um profissional qualificado, capaz de realizar procedimentos clínicos reversíveis, além dos administrativos, denominado auxiliar em saúde bucal (ASB), possibilita uma melhoria na qualidade de vida dos cirurgiões-dentistas, que, por sua vez, refletirá em um modelo de saúde mais amplo para os seus pacientes. Com isso, pretende-se realizar um tratamento odontológico com máxima economia, maior eficiência e menor fadiga para o profissional, para a equipe e para o paciente, substituindo, dessa forma, os processos de trabalho de uma Odontologia estática por condutas racionais, produtivas e atuais [2].

A variávelqualidadede vida tem sido pesquisadaespecialmente com relação a doenças crônicas, no entanto, ainda permanece pouco estudada em populações específicas,como é o caso dos profissionaisda saúde [8]. Levando-se em consideração os componentes geradores do estressepertinentesao exercícioda profissão,a avalia ção da qualidadede vida está se tornandocada vez mais importantem todosos segmentosprofissionaisinclusive na OdontologiaDiantedisso,o objetivodestetrabalhofoi avaliara qualidadede vidade cirugiões-dentistaquetrabalham com e sem ASBs em consultóriosdentáriospar ticulares,mediantea aplicaçãodo questionárioMedical Outcomes Study 36 - Item Short Health Survey (SF-36).

\section{Material E Método}

A qualidade de vida pode ser mensurada por meio de dois grupos de instrumentos, o genérico e o de condição específica. O instrumento selecionado neste estudo foi o Medical Outcomes Study 36 - Item Short Health Survey (SF-36), questionário genérico de avaliação de qualidade de vida, autoaplicávelelaborado por Ware \& Sherboune[9] e validadopara aplicação entre brasileiros por Ciconelli et al. [10].

Esse questionário consta de 36 itens em 11 questões e tem o propósito de avaliar 8 domínios, divididos em dois grandes componentes: o físico - que envolve capacidade funcional, aspectos físicos, dor e estado geral da saúde; e o mental - que abrange saúde mental, aspectos emocionais, sociais e vitalidade, observando que estado geral da saúde e vitalidade estão indiretamente relacionados aos dois segmentos.

A capacidade funcional avalia a presença e a extensão das limitaçõesrelacionadasà capacidade física com três níveis de resposta (muita limitação, pouca ou sem limitação); os aspectos físicos são avaliados pelas limitações físicas na realização do trabalho e das atividades diárias. A dor é pesquisada por meio de sua intensidade, extensão e/ou interferência nas atividades diárias e o estado geral de saúde é avaliado pela autopercepção do respondente. Em relação aos componentes mentais, a vitalidade é avaliada pelo nível de energia e fadiga na realização das atividades da vida diária. Os aspectos sociais são avaliados pela integração do indivíduo às atividades sociais; os emocionais, pelas dificuldades emocionais que limitam o trabalho e as atividades rotineiras; a saúde mental pela interferência da ansiedade, depressão, felicidade, alterações de comportamento ou descontrole emocional e bem-estar psicológico nas atividades da vida diária [11]. Os resultados do SF-36 são mostrados em escores de 0 a 100 obtidos a partir de uma relação de quesitos sobre vários aspectos da qualidade de vida. Quanto maior for o escore, melhor a qualidade de vida do indivíduo [10]. 
O estudo foi do tipo qualitativo-descritivo e a amostra foi constituída de cirurgiões-dentistas que preencheramos requisitospara a inclusão no estudo. Os critériosde inclusãoforam: cirurgiões-dentistasatuando em consultórios particulares, com 10 anos ou mais de exercício profissional, de ambos os gêneros - sendo 30 que trabalhavam auxiliados, na categoria de cirur gião-dentista auxiliado; e 30, sem auxiliar, constituindo a categoria de cirurgiões-dentistasnão auxiliados. Ambas as categorias formaram os subgrupos feminino auxiliado (FA), feminino não auxiliado (FNA), masculino auxiliado (MA) e masculino não auxiliado (MNA). Não fizeram parte deste estudo os cirurgiões-dentistas que, embora tivessem auxiliar odontológico, este não atuava diretamente na sala de clínica, exercendo predominantemente funções administrativas. Cirurgiõesdentistas que exerciam sua função em serviço público também foram excluídos da pesquisa.

Para avaliação dos resultados foram utilizados dois testes de significância estatística,ANOVA e TUKEY, contidosno programaStatisticversão8 (AnalyticalSoft ware, USA, 2003), com nível de significância p $<0,05$.

\section{Resultados}

A influênciados oito domínios(capacidadefuncional - CF; aspecto físico - AF; dor - D; estado geral de saúde - EGS; vitalidade - V; aspectos sociais - AS; aspectos emocionais-AE; saúde mental - SM) sob quatro diferentessubgrupos,classificadosde acordocomo gêneroe com a presençaou não de trabalhoauxiliado(FA, FNA, MA, MNA), sobre a qualidade de vida de cirugiõesdentistasconstituitrintae duas condiçõesexperimentais a seremestudadas $(8 \times 4)$.A estatísticadescritivados oito domínios é apresentada na Figura 1.

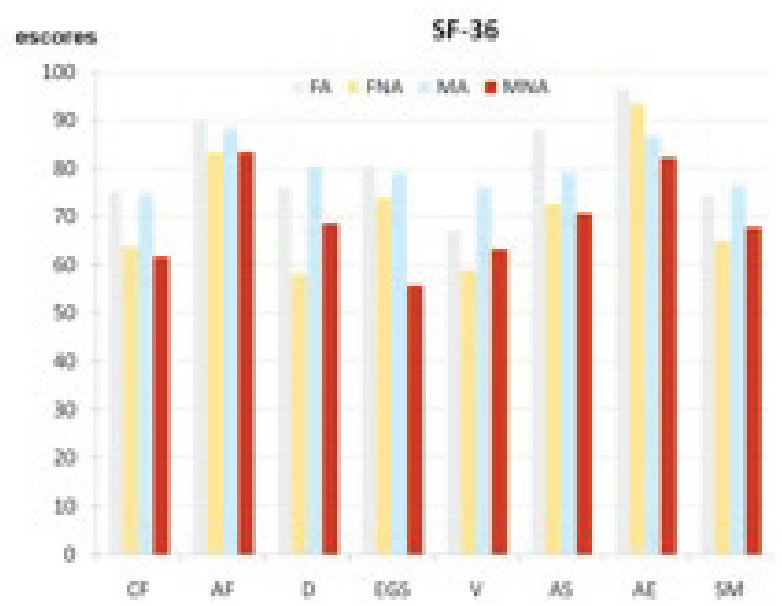

Figura 1 - Gráfico de colunas dos valores médios obtidos para cada subgrupo nos oito diferentes domínios do instrumento SF-36 para avaliação da qualidade de vida.
Os dados encontrados na estatística descritiva permitiram observar que, de modo geral, os maiores escores, obtidos a partir de uma relação de quesitos sobre vários aspectos da qualidade de vida, estão associados à presença de trabalho auxiliado, independente do gênero. Sendo assim, para determinar a influência desses diferentes domínios sobre a qualidade de vida dos cirur giões-dentistas, que trabalham com ou sem ASB, foi aplicado o teste estatístico ANOVA, representado na Tabela 1.

\section{Tabela 1 - Análise estatística dos OITO DOMÍNIOS COM APLICAÇÃO DO TESTE ANOVA.}

\begin{tabular}{c|c}
\hline DOMíNIOS & p-valor \\
\hline Capacidade Funcional & $0,0266^{*}$ \\
\hline Aspecto Físico & 0,8033 \\
\hline D & $0,0075^{\star}$ \\
\hline Estado Geral de Saúde & $0,0019^{\star}$ \\
\hline Vitalidade & $0,0109^{*}$ \\
\hline Aspectos Sociais & $0,0499^{*}$ \\
\hline Aspectos Emocionais & 0,3685 \\
\hline Saúde Mental & 0,0937 \\
\hline
\end{tabular}

${ }^{*} \mathrm{p}<0,05$ - diferença estatisticamente significante

A análise pelo teste ANOV A revelou que os domínios capacidade funcional, dor, estado geral de saúde, vitalidade e aspectos sociais apresentaram diferença estatisticamente significante $(p<0,05)$.

Quando foram comparados os valores dos escores desses domínios específicos, por meio do teste de Tukey (5\%), verificou-se que os as pectos capacidade funcional e dor, em ambos os gêneros, apresentaram maiores valores nos grupos de cirurgiões-dentistas que trabalhavam auxiliados, sendo observada diferença estatis ticamente significante com relação aos grupos não auxiliados. No domínio estado de saúde geral, apenas no grupo masculino foi observada diferen ça estatística. Já no domínio vitalidade, foi obser vada diferença estatística somente entre os grupos masculino auxiliado e feminino não-auxiliado. Por outro lado, com relação aos aspectos sociais, pelo teste de Tukey, essa diferença não foi nitidamente significante ( $\mathrm{T}$ abela 2 ). 
Tabela 2- Capacidade funcional, dor, estado geral de saúde, Vitalidade, aspectos sociais. ResulTADO DO TESTE DE COMPARAÇÃO MÚLTIPLA DE MÉdIAS DE TUKEY (5\%) PARA QUATRO NÍVEIS (SUBGRUPOS).

\begin{tabular}{|c|c|c|c|}
\hline DOMÍNIO & SUBGRUPOS & MÉDIA & DESVIO PADRAO \\
\hline \multirow{4}{*}{$\begin{array}{l}\text { Capacidade } \\
\text { Funcional }\end{array}$} & FA & $75,33^{A}$ & 11,87 \\
\hline & FNA & $64,00^{\mathrm{B}}$ & 16,50 \\
\hline & MA & $74,67^{A}$ & 8,96 \\
\hline & MNA & $61,67^{\mathrm{B}}$ & 20,50 \\
\hline \multirow{4}{*}{$\begin{array}{c}\mathrm{T} \\
\text { Dor }\end{array}$} & FA & $76,13^{A}$ & 20,91 \\
\hline & FNA & $58,00^{B}$ & 16,41 \\
\hline & MA & $80,33^{A}$ & 16,40 \\
\hline & MNA & $68,53^{\mathrm{B}}$ & 18,32 \\
\hline \multirow{4}{*}{$\begin{array}{l}\text { Estado } \\
\text { Geral de } \\
\text { Saúde }\end{array}$} & FA & $80,67^{A}$ & 22,06 \\
\hline & FNA & $73,93^{A}$ & 25,20 \\
\hline & MA & $79,13^{A}$ & 24,09 \\
\hline & MNA & $55,85^{\mathrm{B}}$ & 24,61 \\
\hline \multirow{4}{*}{ Vitalidade } & FA & $67,33^{A B}$ & 14,38 \\
\hline & FNA & $59,00^{\mathrm{B}}$ & 15,61 \\
\hline & MA & $76,00^{A}$ & 9,86 \\
\hline & MNA & $63,33^{A B}$ & 14,96 \\
\hline \multirow{4}{*}{$\begin{array}{l}\text { Aspectos } \\
\text { Sociais }\end{array}$} & FA & $88,33^{A}$ & 17,34 \\
\hline & FNA & $72,50^{A B}$ & 17,80 \\
\hline & MA & $79,17^{\mathrm{AB}}$ & 20,41 \\
\hline & MNA & $70,67^{A B}$ & 18,79 \\
\hline
\end{tabular}

Letras diferentes indicam diferença estatisticamente significante.

\section{Dıscussão}

A expressão "qualidadede vida", como vem sendo aplicadana literatura,não parece ter um único significa do. Tal conceito, de modo geral, refere-se a uma noção eminentementehumana, que tem sido aproximada ao grau de satisfação encontrado na vida familiar, amorosa, social e ambiental,ou à própria estética existencial.No âmbito da saúde, quando visto no sentido ampliado, o conceito se apoia na compreensão das necessidades humanas fundamentais,e, quando visto de forma mais focalizada, coloca sua centralidade na capacidade de viver sem doençasou de superaras dificuldadesdos estadosou condições de morbidade [12].

Quando aplicado a uma população específica, considera-se que o conceito "qualidade de vida" inclua uma variedade potencial de condições que podem afetar a percepção do indivíduo, seus sentimentos e comportamentos relacionados com o seu desempenho diário. Com relação aos profissionais de saúde, mais especificamente os cirurgiões-dentistas, observa-se que a preocupação com relação à qualidade de vida dessa população é pouco explorada, muito embora seja de suma importância no âmbito de saúde pública.

O estresse e outras consequências biopsicofisiológicas às quais esses profissionais estão expostos, de forma cumulativa e progressiva, são desencadeados por inúmeros fatores, dentre eles o ambiente de trabalho, a sobrecarga, as relações interpessoais e o tempo de serviço. Visando aprimorar positivamente tais condições, o conceito de racionalização do trabalho foi desenvolvido, a fim de influenciar não só a qualidade do serviço prestado, mas também a qualidade de vida dos prestadores de tais serviços.

Os estudos de racionalizaçãodo trabalhotêm procut rado dar ao Homem a possibilidadede produzirmais e melhor dentro de uma unidade de tempo; ou, num conceito mais amplo, dar a ele condição de utilizar meios e sistemasquetornemo seu trabalhomaiseficiente,produtivo e menos cansativo[13]. Neste sentido, para que a racionalizaçãodo trabalhopossa ser efetivadaadequada mente em âmbito odontológico, é de suma importância a utilização de pessoal auxiliar qualificado [14].

Dentre as vantagens do trabalho auxiliado na Odontologia, destaca-se o marcante aumento na produtividade, fato este comprovado por vários autores. De 
acordo com dados da literatura científica, a delegação de tarefas ao auxiliar leva o cirurgião-dentista a uma redução de $46 \%$ na prática das funções clínicas, enquanto a inserção de uma segunda auxiliar eleva esse percentual para aproximadamente $62 \%$ [15]. Naressi \& Naressi [13], ao analisar e comparar o desempenho técnico e a produtividade de alunos de Odontologia em ambulatório clínico, concluíram que a produtividade, por aluno, aumentou cerca de $18 \%$ quando trabalhavam no sistema de duplas, caracterizando trabalho auxiliado, em comparação com o trabalho individualizado. Já Orenha et al. [16], ao avaliar a atuação dos auxiliares odontológicos junto aos cirurgiõesdentistas que integravam o serviço público, por meio de entrevistas e de consultas aos mapas de produção, concluíram que a produtividade do grupo auxiliado foi de $112 \%$ maior que a do grupo que trabalhava sem auxiliar. Esses últimos autores verificaram que a principal razão da elevada diferença observada decorria da ocupação de grande parte do tempo profissional dos cirurgiões-dentistas na execução de ações indiretas prévias, simultâneas e complementares, executadas dentro e fora da cavidade bucal, as quais requerem conhecimento técnico de baixa e média complexidade e, por esta razão, deveriam ser executadas por pessoal auxiliar.

Além da melhoria significativa na produtividade, o trabalho auxiliado contribui com outros benefícios, dentre eles a economia de tempo, a continuidade dos movimentos operatórios e o menor desgaste físico e emocional do cirurgião-dentista. Tais fatores poderão influenciar direta ou indiretamente na qualidade de vida desses profissionais $[2,7]$.

Em função disso, esse estudo teve como objetivo avaliar e comparar a qualidade de vida de cirurgiõesdentistas que trabalham com e sem ASBs em consultórios odontológicos particulares, mediante aplicação do instrumento de medida SF-36. Tal estudo mostrou-se relevante, uma vez que as pesquisas têm se voltado predominantemente para a ótica dos pacientes, deixando de lado as condições físicas e mentais dos profissionais por eles responsáveis.

Inicialmente, os profissionais participantes foram classificados com relação à faixa etária e ao tempo de serviço, no intuito de qualificar o perfil dos subgrupos estudados. Da análise dos quatro grupos de cirur giões-dentistas, masculino e feminino, auxiliados e não auxiliados, totalizando 60 profissionais, pode-se verificar que a idade dos cirurgiões-dentistas participantes ficou muito próxima, sendo que a faixa etária média para os subgrupos FA, FNA, MA e MNA foi de $41,8,42,06,45,93$ e 47,86, respectivamente. O tempo de serviço, aferido em anos, também mostrou uma faixa próxima entre os subgrupos analisados; no entanto, os masculinos mostraram um tempo de exercício profissional um pouco maior em relação aos femininos, sendo a média para os subgrupos MA e MNA de 21,13 e 21,4, e FA e FNA de 18,2 e 16,73, respectivamente.

Após a definição do perfil dos subgrupos, os profissionais foram analisados com relação aos fatores associados à qualidade de vida, no intuito de averiguar se o trabalho auxiliado influencia, de alguma maneira, no bem-estar dos cirurgiões-dentistas. A avaliação da qualidade de vida pode ser mensurada por meio de vários instrumentos, dentre eles o questionário SF-36, escolhido como método de análise neste estudo. O SF-36 é um instrumento genérico, multidimensional, de fácil aplicação, confiável e que já foi traduzido, adaptado e validado para a língua portuguesa. Tal questionário é composto de oito domínios: capacidade funcional, aspectos físicos, dor, estado geral de saúde, vitalidade, aspectos sociais, aspectos emocionais e saúde mental. Os resultados do SF-36 são mostrados em escores de 0 a 100, obtidos a partir de uma relação de quesitos sobre vários aspectos da qualidade de vida. Quanto maior for o escore, melhor a qualidade de vida do indivíduo [10].

A análise do domínio capacidade funcional, que avalia a presença e a extensão de limitações relacionadas à capacidade física, apresentou diferença estatisticamente significante; os grupos de cirurgiões-dentistas auxiliados (FA e MA) mostraram uma melhor capacidade funcional em relação aos não auxiliados.

O domínio aspecto físico avalia limitações quanto ao tipo e qualidade de trabalho, bem como quanto essas limitações dificultam a realização do trabalho e das atividades da vida diária. Quando tal domínio foi avaliado, não ocorreram diferenças estatísticas entre os quatro grupos analisados.

Em relação ao domínio que avalia a presença de dor, sua intensidade e sua interferência nas atividades da vida diária, os grupos de cirurgiões-dentistas com auxiliares apresentaram ausência desse estado sintomatológico, diferindo dos grupos sem auxiliares. $\mathrm{O}$ melhor subgrupo foi o MA, seguido pelo FA com valores de 80,33 e 76,13 respectivamente; já os piores grupos foram o FNA e o MNA com valores de 58,00 e 68,53 , respectivamente.

O estado geral de saúde avalia como o indivíduo se sente em relação à sua saúde global. No presente estudo, a presença de trabalho auxiliado, com relação a este domínio específico, foi relevante apenas entre os subgrupos masculinos, sendo observada diferença estatisticamente significante. 
Quanto ao domínio vitalidade,que considerao nível de energia e fadiga do indivíduo, verificou-se que o subgrupo MA apresentou pontuação estatisticamente maior somenteem relaçãoao subgrupoFNA, sugerindo que o fator determinante pode estar no gênero e não na condição de trabalho propriamente dita.

Os aspectos social e emocional não apresentaram variações estatísticas significantes neste estudo, no entanto pode-se analisar pelos resultados que os grupos auxiliados estão levemente melhores do que os grupos não auxiliados, diferença não bem evidenciada talvez em função da não especificidade do instrumento. Com relação ao aspecto emocional, Iida et al. [17] enfatizaram a importância da satisfaçãoe do prazer pessoal. Neste aspecto, uma importante vantagem do trabalho auxiliado é a contribuição para o aumento da segurança e da confiança no paciente, em função da presença de mais um componente na equipe; presença esta cuja atuação permite ao cirurgião-dentista prestar um atendimento de forma mais prazerosa, contribuindo diretamente para o seu bem-estar e qualidade de vida.

O último domínio é o da saúde mental, que inclui questões que avaliam a interferência da ansiedade, da depressão, das alterações no comportamento ou descontrole emocional, assim como o bem-estar psicológico. Os dados obtidos não revelaram variação estatística mostrando que, em relação à saúde mental, todos os cirurgiões-dentistas analisados apresentaram resultados semelhantes.

A amostrautilizadaneste trabalhofoi suficientepara demonstradiferençaentreos subgrupoæscolhidoøuando os domínios capacidade funcional, dor e estado geral de saúde foram analisados. Tais resultados mostraram uma influênciapositivana qualidadede vida dos cirurgiõesdentistas, quando estes trabalham auxiliados.

Valendo-se de diferentes instrumentos, outros autores também estudaram e avaliaram a qualidade de vida na população de cirurgiões-dentistas. Nunes \& Freire [8] realizaram um estudo observacional do tipo transversal, com o objetivo de conhecer a qualidade de vida de cirurgiões-dentistas que atuam em serviço público municipal. Utilizando-se de um questionário contendo o instrumento WHOQOL-Bref da Organização Mundial de Saúde, os autores concluíram que os profissionais pesquisados apresentaram baixa qualidade de vida nos domínios físico e psicológico, que incluíam fatores associados a problemas de saúde, capacidade de trabalho e condições afetivas e cognitivas. Tais resultados, associados aos encontrados no presente estudo, podem ajudar a traçar de maneira mais fidedigna o perfil desses profissionais da saúde.

Revisando a literatura, foi possível observar que, na maior parte dos trabalhos, a preocupação está voltada à qualidade de vida dos pacientes que procuram o tratamento odontológico, deixando em segundo plano as investigações dos fatores que interferem na qualidade de vida do cirurgião-dentista [3]. O presente estudo é um dos poucos que avaliam a qualidade de vida como fator preponderante na classe dos cirurgiões-dentistas. A associação do trabalho auxiliado à qualidade de vida desses profissionais, analisada por meio do instrumento SF-36, até o presente momento, permanece exclusiva a este estudo, dificultando a discussão de resultados que poderiam ter sido encontrados por outros grupos de estudos. Uma vez que o termo "qualidade de vida" vem adquirindo importância ascendente na sociedade contemporânea, principalmente no setor de saúde, incluindo seus profissionais, recomendase que mais estudos sejam realizados nesse sentido, no intuito de aprimorar os conhecimentos e planejar, posteriormente, medidas benéficas dentro deste setor.

\section{Conclusão}

Nos grupos de cirurgiões-dentistas estudados, a condição de trabalho auxiliado impactou positivamente a qualidade de vida considerando os domínios capacidade funcional, dor e estado geral de saúde.

\footnotetext{
ABstract

The use of auxiliary personnel has become a critical point for improving productivity in dental clinics. It directly contributes to reduction of professional stress and fatigue. The objective of this paper is to analyze and discuss the quality of life of dentists (CDs) who work with or without dental assistants in private dental clinics. A measuring tool that has been validated for the Brazilian population, namely the "Medical Outcomes Study 36 - Item Short Health Survey" (SF-36), was used. A questionnaire was developed with 11 questions and 36 items. The purpose was to evaluate each of eight areas that were divided into two major components: the physical (that involves the functional capacity, physical aspects, pain and general state of health) and the mental component (that covers mental health, emotional and social aspects and vitality). The research was of the qualitative-descriptive type and the sample was of $60 \mathrm{CDs}$, men and women, each with at least 10 years of professional experience; 30 out of them worked with assistants and 30 without. The results obtained with the ANOVA and TUKEY tests of statistical significance show statistical variation for functional capacity, pain, general
} 
health conditions, vitality and social aspects. In relation to functional capacity, pain and general health conditions, the study showed a positive impact over the quality of life of the group of dentists (CDs).

\section{UNITERMS}

Odontology; dentistry, assisted work; quality of life; psychosocial impact.

\section{REFERÊNCIAS}

1. WHOQOL Group. WHOQOL: Measuring quality of life. Geneva: WHO; 1997.

2. Garcia PPNS, Terence RL, Souza AC. Avaliação de cirurgiões-dentistas quanto ao uso de pessoal auxiliar na organização do atendimento clínico. Revista de Odontologia da UNESP. 2004;33(1):25-32.

3. Carvalho FS et al. Qualidade de vida do cirurgião-dentista. Rev e Odontol da UNESP. 2008;37(1):65-68.

4. Sales CVMS, Pinto AEA, Cavalcanti AL, Lucas RSCC, Lins SD. Delegação de funções ao pessoal auxiliar odontológico pelos cirurgiões-dentistas da cidade de Campina Grande PB. Rev Ciênc Med Biol. 2007;6(1):47-5.

5. Barros OB. Ergonomia III: auxiliares em odontologia ACD THD - TPD - APD. São Paulo: Pancast; 1995.

6. Pezzato LM, Cocco MIM. O técnico em higiene dental e o atendente de consultório dentário no mundo do trabalho. Saúde em Debate. 2004;28(68):212-19.

7. Paranhos LR, Ricci ID, Tomasso S, Salazar M, Siqueira DF. Análise da relação entre o cirurgião-dentista e o pessoal auxiliar. Rev Odonto Ciênc. 2008;23(4):365-370.

8. Nunes MF, Freire MCM. Qualidade de vida de cirurgiõesdentistas que atuam em um serviço público. Rev Saúde Pública. 2006;40(6):1019-26.

9. Ware JE, Sherbourne CD. The MOS 36-item short form health survey (SF-36): I. Conceptual framework and item selection. Med Care. 1992;30:473-83.

10. Ciconelli RM, Ferraz MB, Santos W, Meinão I, Quaresma MR. Tradução para a língua portuguesa e validação do questionário genérico de avaliação de qualidade de vida SF-36 (Brasil SF-36). Rev Bras Reumatol. 1999;39(3):143-50.

11. Martinez JE. Avaliação da qualidade de vida de pacientes com fibromialgia através do Medical Outcome Survey 36 Item Short-form Study. Rev Bras Reumatol. 1999;39:312-6.

12. Minayo MCS, Hartz ZMA, Buss PM. Qualidade de vida e saúde: um debate necessário. Ciência \& Saúde Coletiva. 2000;5(1):7-18.

13. Naressi SCM, Naressi WG. Estudo comparativo de produtividade entre alunos, trabalhando sós e auxiliados, em ambulatório clínico. Rev Bras Odontol. 1992;49(2):44-7.

14. Saliba TA, Eleutério D, Saliba CA, Moimaz SAS. Trabalho odontológico auxiliado em serviços públicos e particulares. RPG. 1998;5(3):171-6.

15. Pereira AC, Moreira BW. A utilização do auxiliar odontológico para o aumento da produtividade nos serviços públicos. Rev Assoc Paul Cir Dent. 1992;46(5):851-4.

16. Orenha ES, Eleutério D, Saliba NA. Organização do atendimento odontológico no serviço público: trabalho auxiliado, produtividade e ambiente físico. Rev Odontol UNESP. 1998;27(1):215-24.

17. Iida I, Barros T, Sarmet M. Conexão Emocional no Design. In: Design e transversalidade. Moraes D, Krucken L. Belo Horizonte: Santa Clara: Centro de Estudos Teoria, Cultura e Pesquisa em Design. UEMG; 2008. 92 p.
Recebido: 28/04/2011

Aceito: 18/08/2011

Correspondência:

Daniel de Oliveira Leite

Endereço: Rua Uiramirins, $n^{\circ} 160$, casa 110, Jardim Uirá, São José dos Campos CEP: 12227-660 Email: danielolli@yahoo.com.br 